\title{
Control of crystallographic orientation by metal additive manufacturing process of $\beta$-type Ti alloys based on the bone tissue anisotropy
}

\author{
Takayoshi Nakano $^{1 *}$, Takuya Ishimoto $^{1}$, Aira Matsugaki ${ }^{1}$, Koji Hagihara $^{1}$, Yuichiro Koizumi ${ }^{1}$ and Ryosuke Ozasa ${ }^{1}$ \\ ${ }^{1}$ Division of Materials and Manufacturing Science, Graduate School of Engineering; \& Anisotropic Design and Additive Manufacturing \\ Research Center, Osaka University \\ *nakano@mat.eng.osaka-u.ac.jp
}

\begin{abstract}
$\underline{\text { Abstract }}$
Variation in the scanning strategy for $\beta$-type Ti alloys during additive manufacturing (AM) enables the fabrication of a singlecrystal-like microstructure possessing a crystallographic texture, in which the low-Young's modulus- $<100>$ direction is aligned along a specific direction. Thus, metal biomaterial with low elasticity, comparable to the bone Young's modulus, can be developed by AM, which will contribute to suppress the stress shielding of bone and prevent degradation of bone tissue anisotropy.
\end{abstract}

\section{$\underline{\text { 1. Introduction }}$}

$\beta$-type titanium alloys have garnered significant attention as a bone substitute material because of their excellent functions, particularly their low Young's modulus [1-3]. Low elasticity often appears in the unstable $\beta$ region; however, precipitates such as the $\omega-$ phase frequently appears in the region. Recently, our group found a unique dynamic precipitation softening phenomenon in Ti-Nb-base $\beta$-type alloy single crystals [4].

Recently, metal additive manufacturing (AM) has received considerable attention as an attractive process for providing customized and complex outer shapes [5]. Additionally, it enables a microstructure to be controlled through the unique directional distribution of heat flux in each melt pool, repetitive melting/solidification specific to layer-by-layer fabrication, and thermal history [6]. The control of crystallographic texture, which includes the formation of a single crystalline microstructure, is a noteworthy topic in the latest discussions on metal AM [7-10]. Single crystalline materials often exhibit an orientation dependence of mechanical properties such as Young's modulus [2,11,12]; thus, it is possible to select anisotropic or isotropic physical properties according to the requirement. 

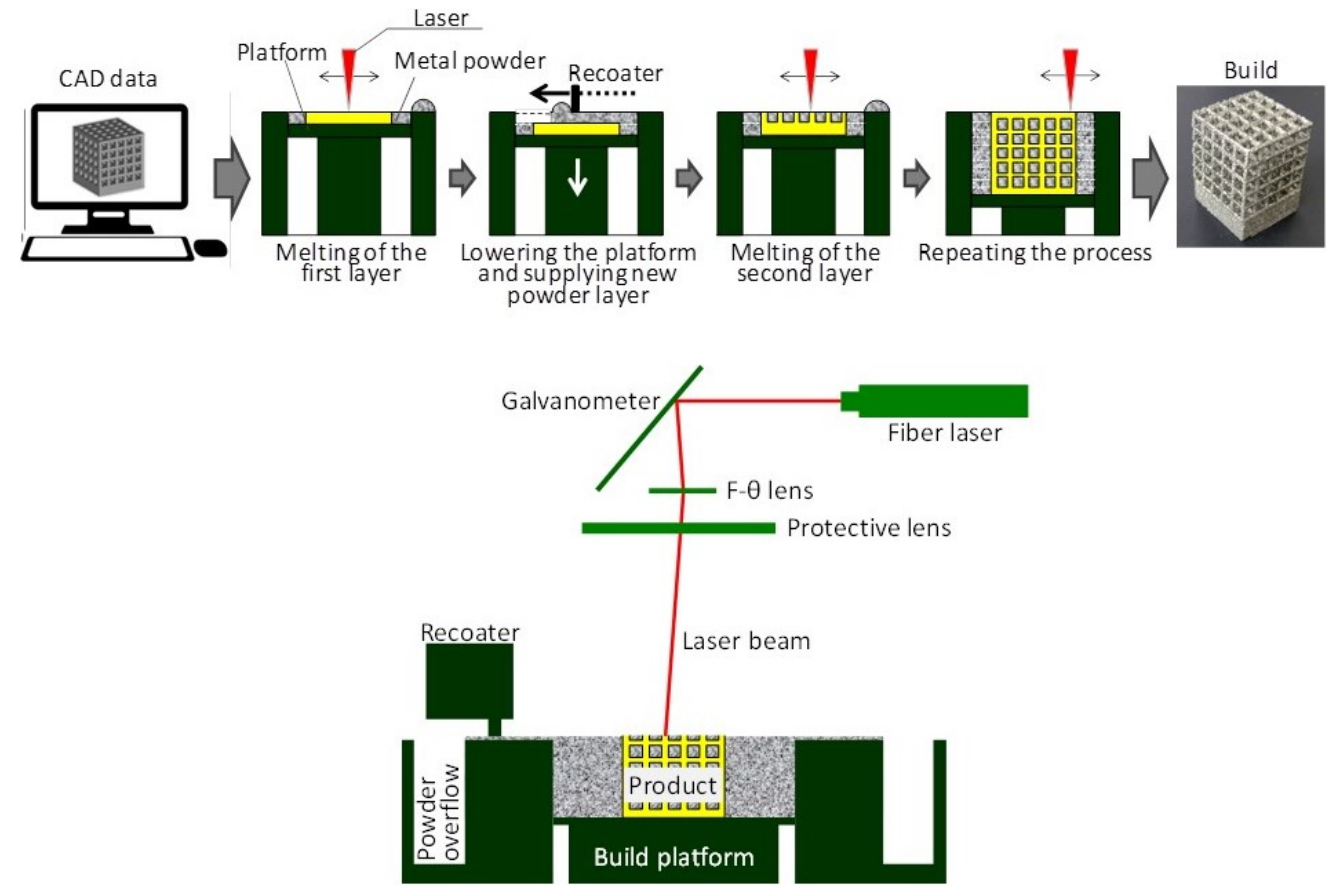

Fig. 1 Schematic illustrations of selective laser beam melting (SLM) method, a kind of a powder bed fusion-type additive manufacturing (AM) process. Reproduced from ref. [5] with modifications.

In this study, therefore, for the fabrication of a $\beta$-type Ti alloy bone substitute, the shape as well as microstructure, especially the crystallographic orientation, were controlled simultaneously via metal AM [13] of the powder bed fusion system, as shown in Fig. 1 [14]. Moreover, bone is composed primarily of collagen fibers and apatite crystals that exhibit three-dimensional preferential orientation as a bone quality parameter depending on the anatomical bone position [15]; thus, the design of a bone substitute should be developed based on the anisotropic bone microstructure [16].

\section{$\underline{\text { 2. Material and experiments }}$}

The texture evolution in $\beta$-type Ti alloy components during fabrication was investigated by selective laser melting (SLM), a type of powder bed fusion process. Two types of scan strategies were utilized for the laser beam. An ingot with a nominal composition of $\mathrm{Ti}-15 \mathrm{Mo}-5 \mathrm{Zr}-3 \mathrm{Al}$ (in mass\%) was used as the starting material. The powder for SLM fabrication was developed by Ar gasatomization. The starting powder was spherical and the median particle size was approximately $80 \mu \mathrm{m}$. Specimens with dimensions of 5 $\mathrm{mm}($ depth) $\times 5 \mathrm{~mm}$ (length) $\times 10 \mathrm{~mm}$ (height) were fabricated in an SLM apparatus (EOS M 290, EOS, Germany) equipped with Yb fiber laser with a laser power, scanning speed, and scan pitch of $360 \mathrm{~W}, 1200 \mathrm{~mm} / \mathrm{s}$, and $100 \mu \mathrm{m}$, respectively. The fabrication was performed in an Ar atmosphere. Two types of scanning strategies were used, as follows: bidirectional (zigzag) scanning in x-axis (Scan 
Strategy_X) and bidirectional scanning with a rotation of $90^{\circ}$ between layers (Scan Strategy_XY). The melt pool morphology after fabrication was observed using an optical microscope (OM; BX60, Olympus, Japan), and inverse pole figures and pole figures were captured using an electron backscatter diffraction (EBSD) system mounted on a field-emission scanning electron microscope (FE-SEM; JSM-6500, JEOL, Japan). After the specimen was cut into cubes of dimensions $5 \mathrm{~mm} \times 5 \mathrm{~mm} \times 5 \mathrm{~mm}$, Young's modulus was analyzed by utilizing a compression test with strain gages. Finally, a bone plate with the combination of Scan Strategies_X and_XY was prototyped in a demonstration.

\section{Results}

$\beta$-type titanium alloys of bec structure exhibit a relatively low Young's modulus even in polycrystalline substances [17-20]. However, in the single crystalline form, they develop an anisotropy of Young's modulus depending on the crystal orientation and exhibit the lowest Young's modulus along $<100>[11,12]$. It is possible to create a bone implant that suppresses stress shielding with a single crystalline material. In addition, the Young's modulus $E_{100}$ and its anisotropy $E_{111} / E_{100}$ depend on the number of valence electrons, $e / a$ and are calculated according to the equations in ref. [11]. As e/a decreases and approaches $4, E_{111} / E_{100}$ increases and $E_{100}$ decreases as shown in Fig. 2 [11].
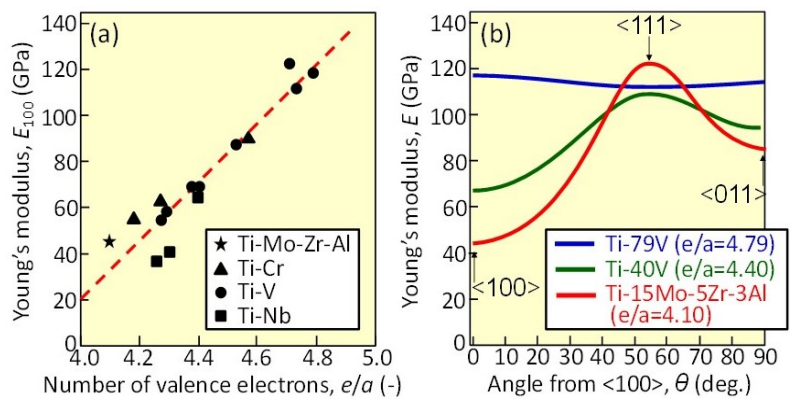

Fig. 2 (a) Young's modulus $E_{100}$ of typical beta-type Ti alloys as a function of $e / a$, and (b) orientation dependence of Young's moduli of Ti-79V, Ti-40V, and Ti-15Mo-5Zr-3Al single crystals. Reproduced from ref. [12] with modifications.

The Ti-15Mo-5Zr-3Al alloy, which exhibits a small $e / a$ of 4.10 and which was approved as a metallic material for biomedical application by ISO (ISO 5832-14) [21], shows a low Young's modulus of approximately $85 \mathrm{GPa}$ in the polycrystalline form, and the $E_{100}$ decreases to $44.4 \mathrm{GPa}$ along $<100>$ in the single crystalline material [11]. This is as low as the modulus of cortical bone $(\sim 30$ $\mathrm{GPa}$ ); hence, placing $<100>$ in parallel with the long axis of long bones may suppress stress shielding. By applying SLM to this alloy and controlling the selectivity of crystal growth orientation with a scan strategy, the crystal orientation can be controlled within the specimens, which enables to align the $<100>$ orientation with a low Young's modulus preferentially along a specific direction of the specimens. 


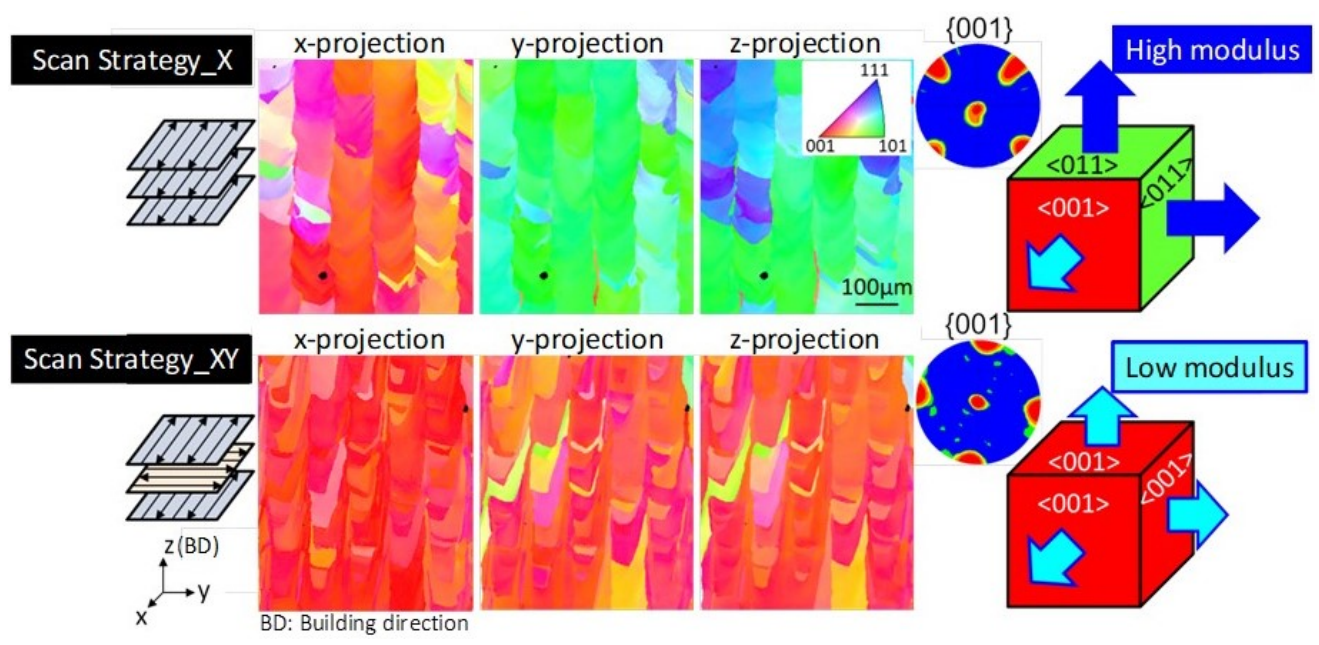

Fig. 3 Inverse pole figure (IPF) images taken in the $y-z$ plane and the corresponding $\{001\}$ and $\{011\}$ pole figures, indicating distinguished crystallographic texture evolution depending on scan strategy [13].

$\beta$-type Ti-15Mo-5Zr-3Al products were fabricated successfully utilizing SLM by tuning the optimal process parameters. Figure 3 shows the crystallographic textures of the fabricated products analyzed by SEM-EBSD in the three orthogonal cross-sections in the products depending on Scan Strategies_X and_XY [13]. The corresponding $\{001\}$ pole figures are also shown in Fig. 3. Completely different textures were developed depending on Scan Strategies. When Scan Strategy_X was applied, strong $<001>$ and $<011>$ alignments occurred along the scanning (x) and building (z) directions, respectively. In contrast, Scan Strategy_XY produced a strong $<001>$ alignment along the $\mathrm{x}, \mathrm{y}$, and $\mathrm{z}$ directions. The obtained results are similar to those reported for other materials with fcc and bcc structures [7-10]. 

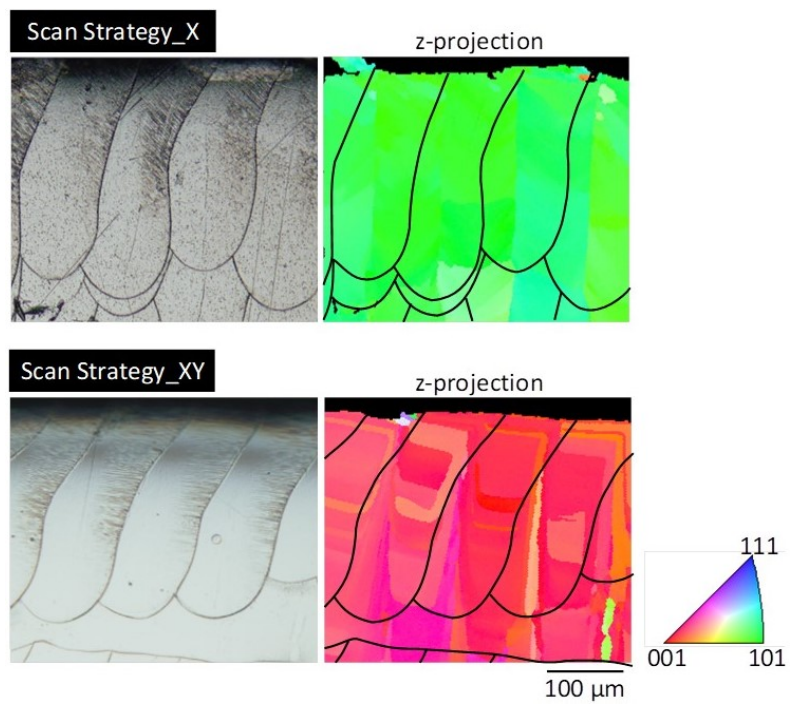

Fig. 4 Optical microscope images and IPF maps captured in the cross-sections of the top of the products ( $y-z$ plane). Melt pool edges are over-written on the IPF maps. Reproduced from ref. [13] with modifications.

Considering the crystallographic texture formation under the layer-by-layer fabrication in the powder bed fusion process, the effect of repetitive heating on the previously solidified position should be considered. The top layer finally deposited during SLM did not undergo subsequent repetitive heating, thus allowing for the clarification of the effect of repetitive heating on the texture. As shown in Fig. 4, the crystallographic orientation in the top layer is consistent with those of the underlying layers, demonstrating that repetitive heating did not change the crystallographic texture. Instead, the texture was found at the point of solidification in the melt pools, thus demonstrating that this crystallographic texture formation was governed by epitaxial crystal growth.

As described above, the control of Young's modulus is strongly desired for the medical application of this alloy as an implant material. The Young's moduli of specimens fabricated in this study were measured along the $\mathrm{x}$ and $\mathrm{y}$ directions. By Scan Strategy_X, the fabricated specimen exhibited a low Young's modulus of approximately $69 \mathrm{GPa}$ in the $<100>$-oriented direction. Moreover, the Young's modulus in the direction in which $<011>$ preferentially orients is approximately $100 \mathrm{GPa}$, representing the ability to realize products with anisotropy in the material parameter through metal AM.

Clearly, a significant anisotropy in the elastic properties was achieved only in the products fabricated by Scan Strategy_X derived from the evolution of different textures; $<001>$ and $<011>$ preferentially oriented along the $\mathrm{x}$ and $\mathrm{y}$ directions, respectively, in the sample fabricated by Scan Strategy_X, while $<001>$ oriented along both $\mathrm{x}$ and y directions in the sample fabricated by Scan Strategy_XY. This demonstrates the effectiveness of the well-organized SLM process for providing Young's moduli in the products. It is noteworthy, however, that the Young's modulus of the textured product was still higher than that of an ideal single crystal along $<001>$, as shown in Fig. 2. A possible reason is the incomplete crystallographic texture formed by SLM currently. The formation of more prominent single crystalline-like textures and an optimum composition control considering the evaporation of light elements will render the low Young's modulus compatible to bones. 


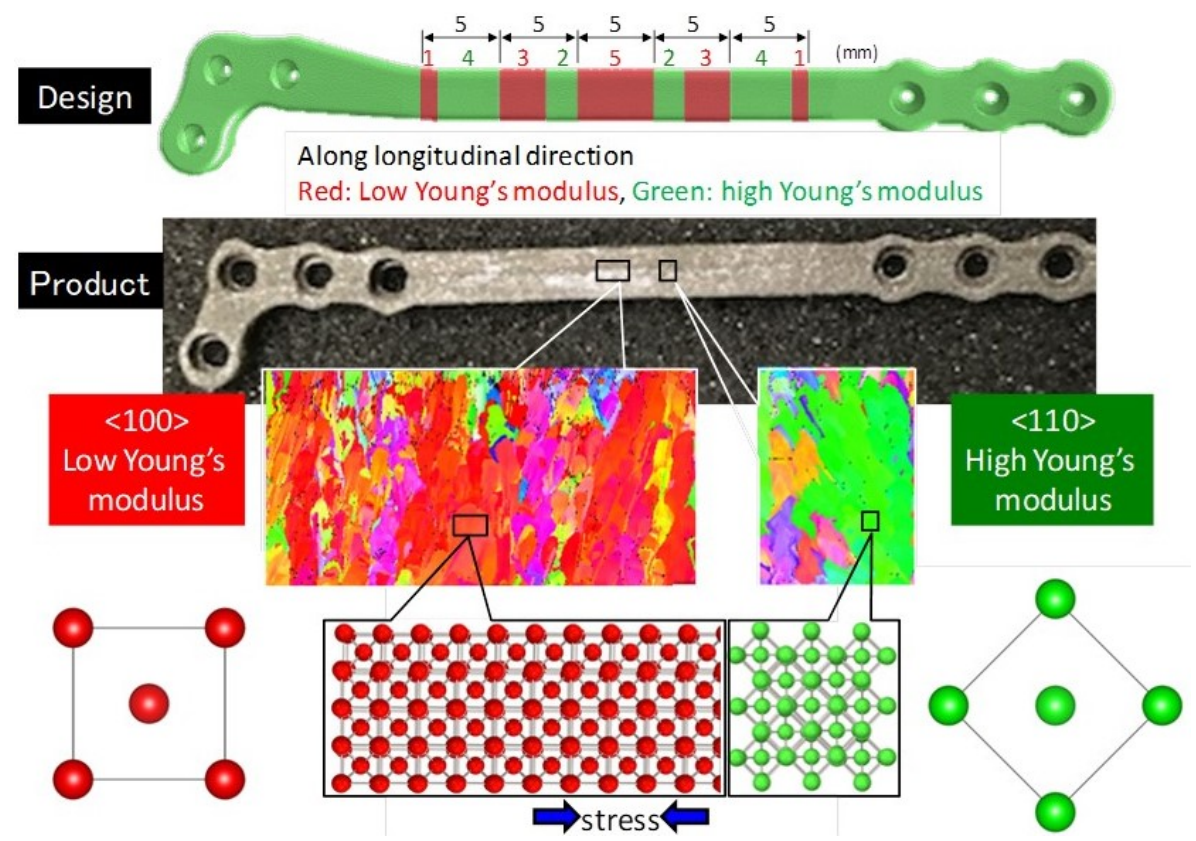

Fig. 5 Trial fabrication of biomedical implant with site-dependently controlled crystallographic orientation and the resultant Young's modulus. The possibility of giving different mechanical properties (Young's moduli) according to the requirements of each region was demonstrated.

With conventional methods, a single crystal with satisfactory shape and size could not be obtained, thus limiting its use as a commercial product. The emergence of metal AM, in which objects of any shape can be fabricated, has increased the expectations to form single crystalline products. Moreover, it is noteworthy that metal AM can flexibly produce different crystallographic textures by different scan strategies, as the above, depending on the position even in the same products. Figure 5 demonstrate the additivemanufactured products composed of different Young's moduli, for the appropriate elastic function in bone implant. A low Young's modulus area shown in red and a relatively high Young's modulus area shown in green correspond to the positions for suppressing the stress shielding of bone and strict fixation, respectively. Furthermore, stress concentration can be avoided by stacking the layers with different Young's moduli. Currently, the patterned crystallographic areas that were almost as designed have been developed, as confirmed by the analyzed crystal orientation map shown in Fig. 5; however, a partially disordered atomic arrangement can be observed owing to the difficulty in the precise control of temperature distribution in narrow areas. This strict control of heat transfer from the surrounding area is challenging to reproduce by computer simulation. Nevertheless, AM technology can enable a methodology that exhibits different functions depending on the material position.

\section{Discussion}


Metal AM is a technique that offers flexible shape control and enables microstructural parameter control through the unique directional distribution of heat flux in each melt pool, repetitive melting/solidification specific to layer-by-layer fabrication, and thermal history. The control of crystallographic texture including the formation of a single crystalline microstructure, is a noteworthy topic in the latest progress on metal AM.

A textured material exhibits an orientation dependence of mechanical properties such as Young's modulus; thus, it is possible to select elastic properties according to the requirement. For example, an anisotropic bone implant that suppresses stress shielding can be achieved by creating texturized materials. Such functionalization by the formation of crystallographic texture can be considered to be a concept similar to the functionalization strategy found in natural structures such as bones.

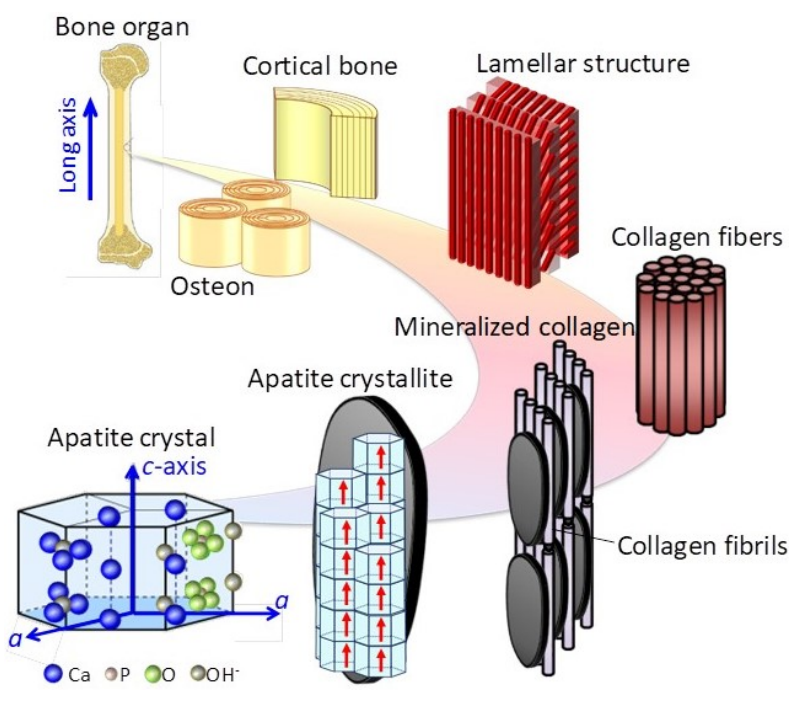

Fig. 6 Schematic representation of the different levels of hierarchical structure of bone: from bone organ to crystal.

The development of biomaterials and medical devices depends on a clear understanding of the mechanical functions, related anisotropic microstructure, and microenvironment of the bone. Bone exhibits a hierarchical multiscale structure [22] and the constituent apatite crystallizes in an anisotropic hexagonal crystal system as an ionic crystal, as shown in Fig. 6. The arrangement of ions is different along the $a$ - and $c$-axes in the apatite crystallite (Fig. 6) [23]. Consequently, various bone functions are expected to vary depending on the crystallographic direction. Moreover, apatite crystallite is distributed along the extending collagen fibril in bone, resulting in enhanced bone anisotropy $[23,24]$. 


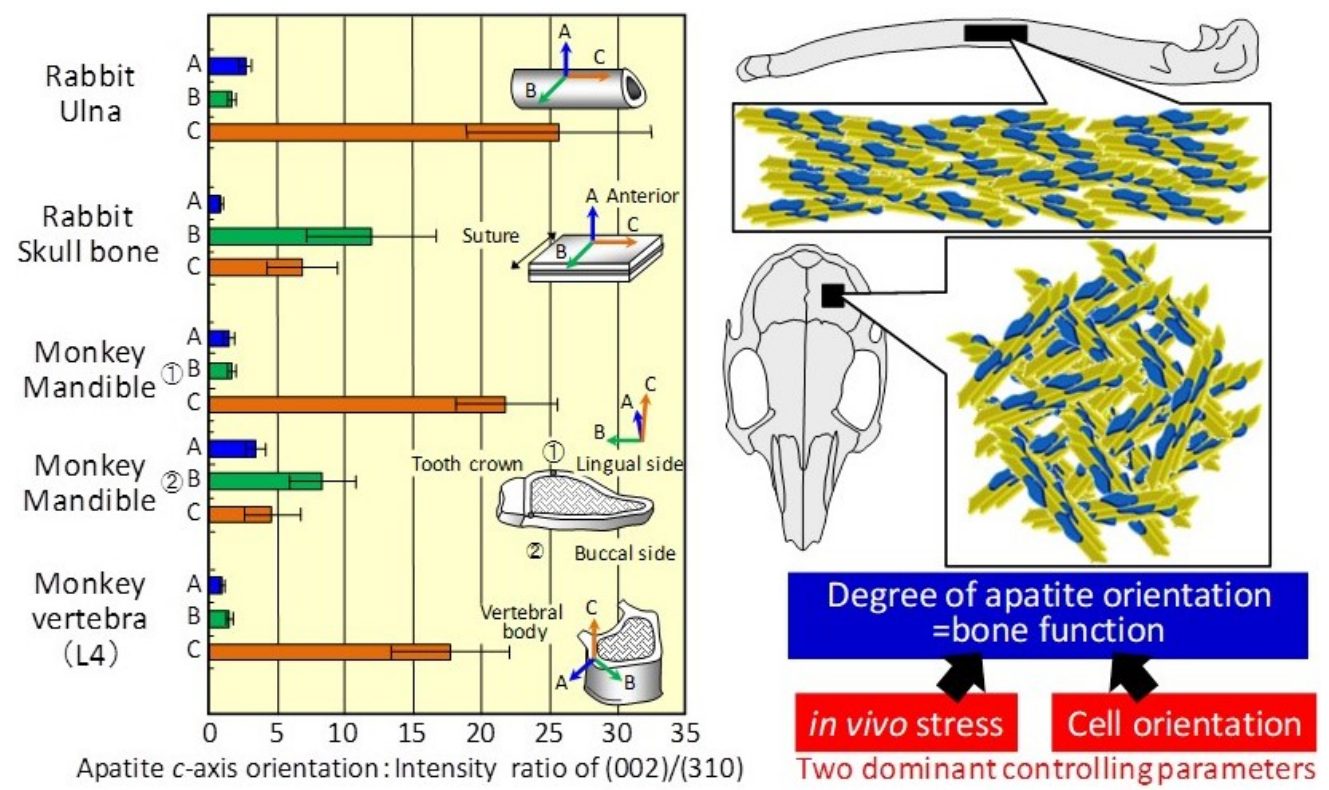

Fig. 7 Distribution of the degree of apatite $c$-axis orientation in various mature cortical bones analyzed using $\mu$ XRD system, and schematic drawings representing one- and two-dimensional orientations. Reproduced from ref. [15] with modifications.

Figure 7 shows the degree of apatite $c$-axis orientation depending on the bone axis and position in various mature cortical bones, analyzed using a micro X-ray diffraction ( $\mu \mathrm{XRD}$ ) system [15]. In a non-orientation case, the relative XRD intensity ratio of $(002) /(310)$ is approximately 2 , indicating that in cases with higher intensity ratios, a preferential orientation of the apatite $c$-axis is shown. In mature cortical bone structures, the bone mineral density (BMD) does not change significantly, but apatite exhibits a onedimensional preferential alignment with the $c$-axis arranged with priority along the bone longitudinal, mesiodistal, and craniocaudal directions in the long bone of rabbit ulna, monkey mandible, and monkey lumbar vertebra, respectively, within the cortical bone portions. Meanwhile, a flat bone from rabbit cranial bone exhibits a two-dimensional orientation along the surface. The characteristic orientation distribution is closely related to the in vivo stress condition, especially the maximum principal stress direction, in bones [15]. Besides the in vivo stress distribution in healthy bone, the degree of anisotropy is sensitive to the pathology, regeneration, and interaction of bone implants, including gene-, molecular-, cellular-, and tissue-related events [22,24,26-36]. Thus, the design of bone implants should be considered in an anisotropic bone tissue microstructure to synchronize the anisotropic atomic arrangement of bone implant and bone tissue anisotropy.

In this study, a unique and useful crystallographic texture can be obtained depending on the scan strategies, for the suppression of stress shielding in bone utilizing the bone implants fabricated by metal AM. A useful crystallographic texture was developed by meltpool-based directional solidification during powder bed fusion because this methodology fabricates products in a track-by-track and layer-by-layer manner by partly remelting the underlying and adjacent parts previously deposited by generating the melt pool. The optimal AM process parameters for controlling the shape and anisotropic crystallographic orientation must be dominated by melt-pool behaviors such as thermal gradient and migration rate of solid/liquid interface based on the energy density, heat transfer, etc. 

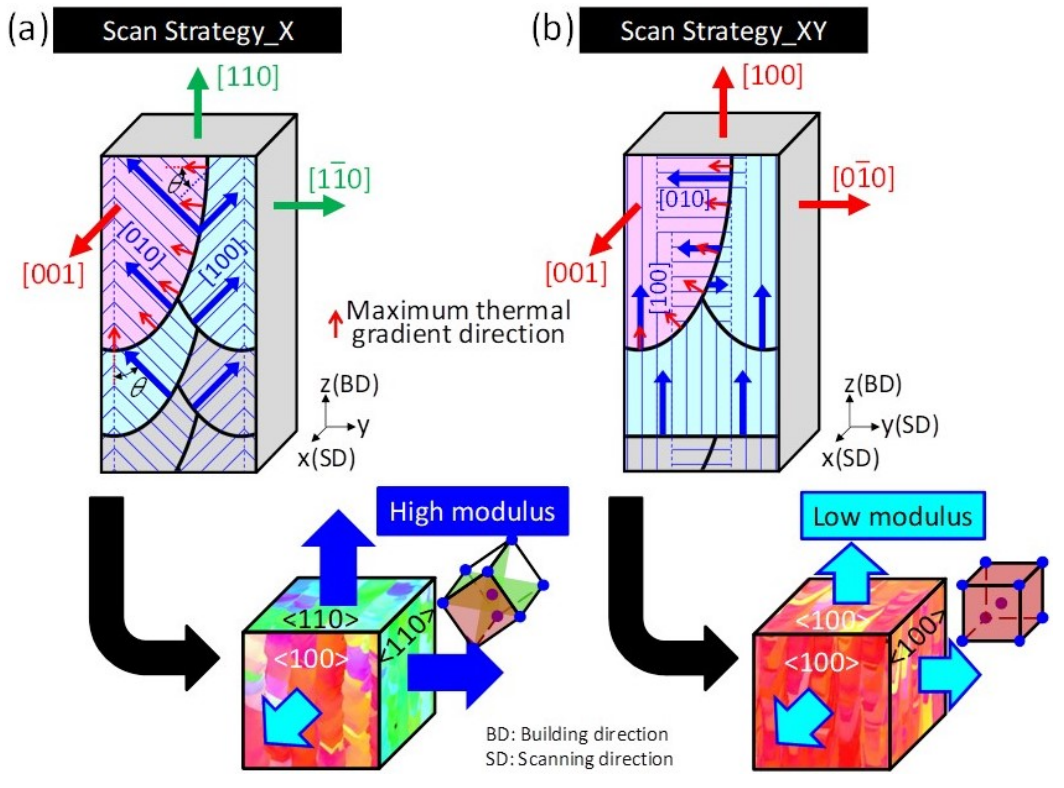

Fig. 8 Relationships among thermal gradient direction (solid/liquid interface migration direction), cellular growth direction, crystallographic orientation direction of substrate, and newly-evolved orientation in a melt pool under Scan Strategies_X and_XY.

Figure 8 schematically summarizes the melt-pool-based mechanism of crystallographic texture evolution. The development of cellular microstructure occurs predominantly in the plane perpendicular to the scanning direction in the melt pool under the present SLM process conditions [13]. The maximum thermal gradient is directed toward the center of the ellipse, vertical to the melt pool edge, in a single melt pool, as indicated by red arrows in the figure. For fabrication with Scan Strategy_X, a $<011>$ preferential orientation along the building direction $(\mathrm{z})$ is caused by two-directional $<001>$-oriented cellular growths at angles of $-45^{\circ}$ and $45^{\circ}$ from the building direction, represented by blue arrows, within the y-z plane in melt pool, as illustrated in Fig. 8(a). By contrast, for fabrication with Scan Strategy_XY, $<001>$ is preferentially oriented along the building direction, owing to the two-directional $<001>$ epitaxial growths that are $0^{\circ}$ and $90^{\circ}$ from the building direction, as illustrated in Fig. 8(b). In this initial stage, the scanning direction along $<001>$ overlaps on the underlying layer along $<011>$, resulting in the growth of $<001>$ prior to that of $<011>$ for Scan Strategy_XY. In the middle stage and thereafter, epitaxial growth in the crystal orientation becomes dominant independent of Scan Strategies_X and _XY, as shown in Fig. 4. Such control of crystal orientation based on scan strategy yields site-dependent patterning with optimal function.

\section{Conclusion}


As described, metal AM is an extremely effective method for the crystallographic texture control of titanium alloys, especially $\beta$-Ti alloys. Metal AM exhibiting the capacities for both shape and microstructural (crystallographic orientation) control is expected to contribute primarily to the creation of products with sophisticated functions. Instead of being limited to the control of shapes, metal AM allows for the control of the material parameter, thus rendering it a promising technology for highlighting the ultimate properties of metallic materials. As a new processing technology, AM is highly compatible with titanium, exhibiting high reactivity and requiring custom-made production. Therefore, it is expected to become a leading step in titanium processing.

Finally, regenerative medicine and the curing of bone diseases should be performed based on the anisotropic bone microstructure. The anisotropic-complicated microstructures of bone tissue, therefore, should always be considered in the development of bone-related biomaterials and medical devices for possible AM applications.

\section{$\underline{\text { Acknowledgments }}$}

This work was supported by the Grants-in-Aid for Scientific Research (S) (JP18H05254) from the Japan Society for the Promotion of Science (JSPS), Cross-Ministerial Strategic Innovation Promotion Program (SIP) from New Energy and Industrial Technology Development Organization (NEDO), and Japan Science and Technology Agency (JST).

\section{$\underline{\text { References }}$}

[1] M. Niinomi, J. Mech. Behav. Biomed. Mater. 1 (2008), 30-42.

[2] S.-H. Lee, K. Hagihara, T. Nakano, Metal. Mater. Trans. A 43 (2012) 1588-1597.

[3] M. Tane, S. Akita, T. Nakano, K. Hagihara, Y. Umakoshi, M. Niinomi, H. Mori, H. Nakajima, Acta Mater. 58 (2010) $6790-6798$.

[4] K. Hagihara, T. Nakano, M. Todai, Sci. Rep. 7 (2017) srep8056.

[5] T. Nakano, J. Jpn. Inst. Light Metals 67 (2017) 470-480.

[6] M. Todai, T. Nakano, T. Liu, H. Y. Yasuda, K. Hagihara, K. Cho, M. Ueda, M. Takayama, Addit. Manufact. 13C (2017) 61-70.

[7] T. Nagase, T. Hori, M. Todai, S.-H. Sun, T. Nakano, Mater. Design 173 (2019) 107771.

[8] S.-H. Sun, T. Ishimoto, K. Hagihara, Y. Tsutsumi, T. Hanawa, T. Nakano, Scripta Mater. 159 (2018) 89-93.

[9] S.-H. Sun, K. Hagihara, T. Nakano, Mater. Design 140 (2018) 307-316.

[10] K. Hagihara, T. Nakano, M. Suzuki, T. Ishimoto, Suyalatu, S.-H. Sun, J. Alloys Compd. 696 (2017) 67-72.

[11] S.-H. Lee, M. Todai, M. Tane, K Hagihara, H. Nakajima, T. Nakano, J. Mech. Behav. Biomed. Mater. 14 (2012) $48-54$.

[12] M. Tane, S. Akita, T Nakano, K. Hagihara, Y. Umakoshi, M. Niinomi, H. Nakajima, Acta Mater. 56 (2008) $2856-2863$.

[13] T. Ishimoto, K. Hagihara, K. Hisamoto, S.-H. Sun, T. Nakano, Scripta Mater. 132 (2017) 34-38. 
[14] T. Nakano, T. Ishimoto, KONA Powder Particle J. 32 (2015) 75-84.

[15] T. Nakano, K. Kaibara, Y. Tabata, N. Nagata, S. Enomoto, E. Marukawa, Y. Umakoshi, Bone 31 (2002) 479-487.

[16] T. Nakano, Bone Tissue and Biomaterial Design Based on the Anisotropic Microstructure, Advances in Metallic Biomaterials, ed.

M. Niinomi, T. Narushima, M. Nakai, Springer (2015) 3-30.

[17] K. Hagihara, T. Nakano, H. Maki, Y. Umakoshi, M. Niinomi, Sci. Rep. 6 (2016) srep29779.

[18] M. Tane, T. Nakano, S. Kuramoto, M. Hata, M. Niinomi, N. Takesue, T. Yano, H. Nakajima, Acta Mater. 59 (2011) $6975-6988$.

[19] M. Todai, T. Nagase, T. Hori, A. Matsugaki, A. Sekita, T. Nakano, Scripta Mater. 129C (2017) 65-68.

[20] T. Nagase, M. Todai, T. Hori, T. Nakano, J. Alloys Compd. 753 (2018) 412-421.

[21] ISO 5832-14:2019, Implants for surgery -- Metallic materials -- Part 14: Wrought titanium 15-molybdenum 5-zirconium 3aluminium alloy, (2019).

[22] A. Sekita, A. Matsugaki, T. Nakano, Bone 97 (2017) 83-93.

[23] J.C. Elliott, Structure and Chemistry of the Apatites and Other Calcium Orthophosphates, Elsevier, (1994).

[24] R. Ozasa, T. Ishimoto, S. Miyabe, J. Hashimoto, M. Hirao, H. Yoshikawa, T. Nakano, Calcif. Tissue Int. 104, (2019) $449-460$.

[25] W.J. Landis, Bone 16 (1995) 533-544.

[26] Y. Nakanishi, A. Matsugaki, K. Kawahara, T. Ninomiya, H. Sawada, T. Nakano, Biomaterials 209 (2019) $103-110$.

[27] A. Matsugaki, T. Harada, Y. Kimura, A. Sekita, T. Nakano, Int. J. Mol. Sci. 19 (2018) 3474.

[28] R. Ozasa, A. Matsugaki, Y. Isobe, T. Saku, H.-S. Yun, T. Nakano, J. Biomed. Mater. Res. A 106 (2018) 360-369.

[29] T. Ishimoto, B. Sato, J. -W. Lee, T. Nakano, Bone 103 (2017) 216-223.

[30] J. -W. Lee, A. Kobayashi, T. Nakano, J. Bone Miner. Metab. 35 (2017) 308-314.

[31] Y. Kimura, A. Matsugaki, A. Sekita, T. Nakano, Sci. Rep. 7 (2017) srep44824.

[32] A. Sekita; A. Matsugaki, T. Ishimoto, T. Nakano, J. Struct. Biol. 197 (2016) 260-270.

[33] J.-W., Lee, H.-S., Yun, T. Nakano, Tissue Eng. C 22 (2016) 856-863.

[34] A. Matsugaki, G. Aramoto, T. Ninomiya, H. Sawada, S. Hata, T. Nakano, Biomaterials 37 (2015) 134-143.

[35] T. Ishimoto, T. Nakano. Y. Umakoshi, M. Yamamoto, Y. Tabata, J. Bone Miner. Res. 28 (2013) 1170-1179.

[36] Y. Noyama, T. Nakano, T. Ishimoto, T. Sakai, H. Yoshikawa, Bone 52 (2013) 659-667. 